\title{
Tract-Based Spatial Statistics of Magnetic Resonance Images to Assess Disease and Treatment Effects in Perinatal Asphyxial Encephalopathy
}

\author{
EMMA J. PORTER, SERENA J. COUNSELL, A. DAVID EDWARDS, JOANNA ALLSOP, AND DENIS AZZOPARDI
}

Institute of Clinical Sciences, MRC Clinical Sciences Centre, Hammersmith Campus, Imperial College London, London W12 ONN, United Kingdom

\begin{abstract}
Biomarkers are required for efficient trials of neuroprotective interventions after perinatal asphyxia. This study aimed to determine whether diffusion tensor imaging (DTI) analyzed by tractbased spatial statistics (TBSS) may be a suitable biomarker of disease and treatment effects after perinatal asphyxia in small groups of patients. We performed TBSS from DTI obtained at $3 \mathrm{~T}$ from eight healthy control infants, 10 untreated and 10 hypothermia-treated infants with neonatal encephalopathy. Median (range) postnatal age at scan was $1 \mathrm{~d}(1-21)$ in the healthy infants, $6 \mathrm{~d}(4-20)$ in the cooled, and $7 \mathrm{~d}(4-18)$ in noncooled infants. Compared with the control group, fractional anisotropy (FA) was significantly reduced not only in several white matter tracts in the noncooled infants but also in the internal capsule in the cooled group. Noncooled infants had significantly lower FA than the cooled treated infants, indicating more extensive damage, in the anterior and posterior limbs of the internal capsule, the corpus callosum, and optic radiations. We conclude that perinatal hypoxic ischemic encephalopathy is associated with widespread white matter abnormalities that are reduced by moderate hypothermia. DTI analyzed by TBSS detects this treatment effect and is therefore a qualified biomarker for the early evaluation of neuroprotective interventions. (Pediatr Res 68: 205-209, 2010)
\end{abstract}

$\mathrm{P}$ erinatal hypoxic ischemic encephalopathy is a major cause of perinatal mortality and morbidity and accounts for $\sim 20 \%$ of all cases of cerebral palsy. Death or major disability occurs in $\sim 60 \%$ of infants with moderate to severe encephalopathy (1). However, recent studies have shown that treatment with moderate hypothermia after resuscitation increases survival without neurologic deficit at the age of $18 \mathrm{mo}$, providing proof of concept that neural rescue therapy applied after resuscitation from asphyxia is possible (2-4).

In experimental studies, a large number of other interventions also have neuroprotective effects after hypoxiaischemia, and some may have synergistic effects when added to cooling (5). To reduce death and disability further after perinatal asphyxia, these laboratory observations need to be translated into early phase "first in infant" trials of small groups of human infants to demonstrate biologic effects and to prepare for pragmatic trials of successful candidates in larger populations. However, biomarker out-

Received March 18, 2010; accepted May 6, 2010.

Correspondence: Denis Azzopardi, M.D., F.R.C.P.C.H., Division of Neonatology, Hammersmith Hospital, Du Cane Road, 5th Floor Ham House, London W12 OHS, United Kingdom; e-mail: d.azzopardi@imperial.ac.uk

Supported by the Imperial College Healthcare Biomedical Research Centre. comes are needed for such early phase studies, and there is currently a paucity of biomarkers qualified to detect treatment effects after asphyxia.

Magnetic resonance imaging (MRI) provides valuable information in infants with hypoxic ischemic encephalopathy: it has substantial reproducibility and accuracy when compared with postmortem histologic appearance and is an accurate early predictor of poor neurologic outcome $(6-10)$. Visual assessment of MRI in a large subgroup of 131 infants participating in a randomized trial of moderate hypothermia showed a reduction of cerebral lesions after moderate hypothermia, which correlated with neurologic outcome (11). Thus, MRI seems to detect the treatment effect of cooling and is a promising candidate biomarker. However, it has not yet been shown to detect treatment effects in small samples suitable for early phase studies of new therapies.

Diffusion tensor imaging (DTI) uses the anisotropic properties of water diffusion to provide a quantitative, reproducible measure of tissue microstructure. Water diffuses more easily along the major axis of a white matter fiber bundle than perpendicular to it. DTI uses this phenomenon to characterize the 3D spatial distribution of water diffusion in each MRI voxel $(12,13)$. Brain tissue diffusion is affected by the local tissue microstructure, and DTI has emerged particularly as a sensitive imaging modality for examining brain injury in hypoxic ischemic encephalopathy. One of the measures most commonly derived from diffusion data is fractional anisotropy (FA), which quantifies directional coherence in local tract structure. FA decreases if the local tract structure is disrupted, reflecting tissue damage (14-17). Previous DTI studies have all used a region of interest (ROI)-based approach, which is time consuming and potentially introduces observer error. However, a recently described technique, tract-based spatial statistics (TBSS) is an automated groupwise observer-independent whole-brain approach that analyses aligned FA images from multiple subjects to allow statistically powerful comparisons of DTI without the need for subjectively defined regions or features of interest (18).

\footnotetext{
Abbreviations: DTI, diffusion tensor imaging; FA, fractional anisotropy; MR, magnetic resonance; ROI, region of interest; TBSS, tract based spatial statistics
} 
These characteristics suggested that TBSS may improve the accuracy of DTI to produce a powerful imaging biomarker for assessing neuroprotective interventions in small groups of patients. To test this hypothesis, we used TBSS to compare FA in a control group of healthy infants and small subgroups of infants suffering moderate to severe hypoxic ischemic encephalopathy who had participated in the moderate hypothermia to treat perinatal asphyxial encephalopathy trial, the TOBY trial (4).

\section{PATIENTS AND METHODS}

This retrospective cohort study examined MRI scans of term infants from our institution participating in the TOBY trial (ISRCTN 89547571) from 2002 to 2006 because of a diagnosis of hypoxic ischemic encephalopathy. We included all infants who were scanned at $3 \mathrm{~T}$ with DTI obtained in 15 noncollinear directions within the first $21 \mathrm{~d}$ of life. The TOBY trial was approved by the London Multicenter Research Ethics Committee, and an additional approval for carrying out the MRI scans at $3 \mathrm{~T}$ was granted by the Hammersmith Hospitals Trust Research Ethics Committee.

Infants were evaluated for entry into the TOBY trial according to a three-step eligibility system based on clinical, neurologic, and EEG criteria, as reported previously (4). After the written parental consent, infants were randomized to moderate systemic hypothermia (rectal temperature $33-34^{\circ} \mathrm{C}$ ) maintained using a cooling mattress for $72 \mathrm{~h}$ starting within the 6th postnatal hour $(n=10)$ or to normothermia $(n=10)$, with rectal temperature maintained at $37 \pm 0.2^{\circ} \mathrm{C}$. All infants received similar standard intensive care. We also examined eight, term control healthy newborns born after a normal pregnancy and delivery.

Furthermore, written parental consent was obtained before imaging for each subject. MRI was performed on a Philips 3T scanner (Philips Medical Systems, The Netherlands) using a phased array head coil. 3D MPRAGE imaging and high-resolution T2-weighted fast spin echo were obtained before DTI. Single shot echo planar DTI was acquired in 15 noncollinear directions using the following parameters: TR $8000 \mathrm{~ms}$, TE $49 \mathrm{~ms}$, slice thickness $2 \mathrm{~mm}$, field of view $224 \mathrm{~mm}$, matrix $128 \times 128$ (voxel size $=1.75 \times 1.75 \times 2$ $\mathrm{mm}^{3}$ ), and $b=750 \mathrm{~s} / \mathrm{mm}^{2}$. The data were acquired with a SENSE factor of 2. This sequence takes $5 \mathrm{~min}$.

Pulse oximetry was performed on all infants throughout the MRI examination. The infants with encephalopathy also had electrocardiographic monitoring throughout each scan. Ear protection was used for each infant, comprising individually molded earplugs using silicone-based dental putty (President Putty; Coltene/Whaledent, Mahwah, NJ) that was placed in the external ear and neonatal earmuffs (Natus MiniMuffs; Natus Medical Inc., San Carlos, CA). The HIE infants were scanned after sedation with oral chloral hydrate $(25-50 \mathrm{mg} / \mathrm{kg})$. The term born controls were imaged after a feed during natural sleep. All examinations were supervised by a pediatrician experienced in MRI procedures.

Image analysis. Data analysis was performed using FSL software (www.fmrib.ox.ac.uk/fsl/). Diffusion tensor images were registered to the nondiffusion volume using affine transformations to reduce distortions because of eddy currents, and a brain extraction process was used to remove any extra cerebral tissue. FA maps were generated using FDT (www.fmrib.ox.ac.uk/fs1/). Voxelwise statistical analysis of FA maps was performed using TBSS (18). FA images from all the subjects in the groups to be compared were aligned to a common space using nonlinear registration. The mean FA image was created and "thinned" to generate a mean FA skeleton that represented the centers of all tracts common to the group. The threshold was set to FA $\geq 0.15$. This is lower than the preset threshold of $\geq 0.2$ as recommended by Smith et al. (18) to take into account the reduced FA in the injured brain but still include major white matter pathways and exclude areas of low FA including peripheral small tracts, where there may be high intersubject variability and gray matter. Each subject's aligned FA image was then projected on to the skeleton, and these FA data were used in voxelwise cross-subjects analyses fully corrected for multiple comparisons across space using threshold free cluster enhancement, with $p<0.05$ considered significant. This process is fully automated and does not require any operator input once initiated; nevertheless image analysis was performed by researchers blind to the subject groupings. The data were also corrected for the postmenstrual age at scan so that all results were independent of this factor.
To explore the data further, we selected the voxels that were identified as being significantly different by TBSS in each tract and calculated the mean FA values for each baby. This allowed us to graphically show and confirm the differences in mean FAs for individual tracts between the groups detected by TBSS. However, we did not perform further statistical analysis of these selective data.

\section{RESULTS}

The clinical characteristics of the study infants are shown in Table 1 . There were no significant differences in the clinical characteristics between the cooled and noncooled infants. The median (range) postnatal age at scan was $1 \mathrm{~d}(1-21)$ in the healthy infants, $6 \mathrm{~d}(4-20)$ in the cooled, and $7 \mathrm{~d}(4-18)$ in noncooled infants $(p>0.05)$.

Compared with the healthy control group, the noncooled group of infants with hypoxic ischemic encephalopathy had significantly reduced FA in many white matter tracts throughout the brain: bilaterally in the anterior and posterior limb of the internal capsule; throughout the external capsule; the body of the corpus callosum; the cingulum; the fornix; the superior longitudinal fasciculus and the superior corona radiata; and the inferior longitudinal fasciculus on the left side (Fig. 1). When the cooled group of infants was compared with the control group, FA was significantly reduced only in the internal capsule (Fig. 2).

While comparing the cooled and noncooled infants with encephalopathy, significantly reduced FA was found in the noncooled compared with the cooled infants in several white matter tracts, including the superior corona radiate, corpus callosum, internal capsule, external capsule, and optic radiations (Fig. 3). The mean FA values for the cooled and noncooled hypoxic ischemic encephalopathy groups are presented in Table 2 and Fig. 4.

\section{DISCUSSION}

MRI is the best imaging modality for assessing the severity of brain injury and long-term outcome for full-term asphyxiated infants. Conventional MRI has allowed better understanding of the timing and patterns of injury that follow perinatal asphyxia. After birth asphyxia, abnormalities on MRI are most commonly observed in the basal ganglia and thalami and in specific white matter areas, namely the posterior limb of the internal capsule; when examined in a large group of 131 infants, these characteristic abnormalities were reduced by therapeutic hypothermia and visual analysis of MRI accurately predicted neurologic outcome at the age of 18 mo, suggesting that MRI detects tissue damage accurately and provides a qualified biomarker for therapeutic interventions after asphyxia when relatively large numbers of subjects are studied (11). MRI spectroscopy has also been shown to predict outcome accurately after asphyxia in similar-sized human studies and to detect the effects of treatment in animal studies (19-21).

However, the aim of this study was to examine the value of MRI as a biomarker for smaller numbers of patients and thus its suitability in early phase "first in infant" trials. DTI is a powerful technique for investigating cerebral microstructure. Diffusion characteristics change rapidly after injury and quan- 
Table 1. Clinical characteristics of study infants

\begin{tabular}{|c|c|c|c|c|c|c|}
\hline & Sex & GA & Birth weight & GA at scan & Age at scan (d) & Amplitude integrated EEG \\
\hline Term control 1 & $\mathrm{M}$ & $38+1$ & 4.30 & $38+2$ & 1 & Not done \\
\hline Term control 2 & $\mathrm{~F}$ & $38+4$ & 3.02 & $39+0$ & 3 & Not done \\
\hline Term control 3 & M & $39+0$ & 2.99 & $39+1$ & 1 & Not done \\
\hline Term control 4 & $\mathrm{~F}$ & $39+2$ & 3.81 & $39+3$ & 1 & Not done \\
\hline Term control 5 & $\mathrm{~F}$ & $40+6$ & 4.00 & $40+6$ & 1 & Not done \\
\hline Term control 6 & M & $41+0$ & 4.03 & $41+1$ & 1 & Not done \\
\hline Term control 7 & $\mathrm{~F}$ & $39+0$ & 3.60 & $41+4$ & 18 & Not done \\
\hline Term control 8 & M & $39+0$ & 3.31 & $42+0$ & 21 & Not done \\
\hline Noncooled 1 & $\mathrm{~F}$ & $41+0$ & 3.34 & $41+6$ & 6 & Severe/low voltage \\
\hline Noncooled 2 & M & $37+3$ & 2.78 & $38+5$ & 9 & Moderate/burst suppression \\
\hline Noncooled 3 & M & $39+0$ & 3.23 & $39+6$ & 6 & Moderate/burst suppression \\
\hline Noncooled 4 & $\mathrm{~F}$ & $40+4$ & 3.73 & $41+6$ & 9 & Severe/low voltage \\
\hline Noncooled 5 & $\mathrm{~F}$ & $40+0$ & 2.76 & $40+4$ & 4 & Severe/low voltage \\
\hline Noncooled 6 & M & $40+3$ & 4.30 & $41+2$ & 6 & Severe/seizures/low voltage \\
\hline Noncooled 7 & $\mathrm{~F}$ & $40+5$ & 3.79 & $41+6$ & 8 & Severe/low voltage \\
\hline Noncooled 8 & $\mathrm{~F}$ & $39+0$ & 3.26 & $42+1$ & 15 & Moderate/burst suppression \\
\hline Noncooled 9 & M & $40+2$ & 3.39 & $41+1$ & 6 & Severe/low voltage \\
\hline Noncooled 10 & M & $41+0$ & 3.60 & $43+4$ & 18 & Severe/burst suppression \\
\hline Cooled 1 & M & $41+5$ & 5.10 & $43+2$ & 11 & Burst suppression \\
\hline Cooled 2 & M & $41+5$ & 2.80 & $42+5$ & 4 & Severe/seizures \\
\hline Cooled 3 & M & $41+6$ & 4.09 & $42+5$ & 6 & Severe/seizures \\
\hline Cooled 4 & $\mathrm{~F}$ & $41+0$ & 3.17 & $41+5$ & 5 & Burst suppression/severe + seizures \\
\hline Cooled 5 & M & $41+4$ & 3.79 & $42+3$ & 6 & Low voltage/severe \\
\hline Cooled 6 & M & $41+4$ & 3.65 & $42+5$ & 8 & Low voltage/severe \\
\hline Cooled 7 & M & $41+4$ & 3.76 & $42+1$ & 4 & Low voltage/severe \\
\hline Cooled 8 & M & $38+0$ & 3.17 & $38+6$ & 6 & Normal/mild + seizures \\
\hline Cooled 9 & $\mathrm{~F}$ & $39+2$ & 3.54 & $40+0$ & 5 & Burst suppression/severe \\
\hline Cooled 10 & M & $40+0$ & 3.72 & $42+6$ & 20 & Low voltage/severe \\
\hline
\end{tabular}
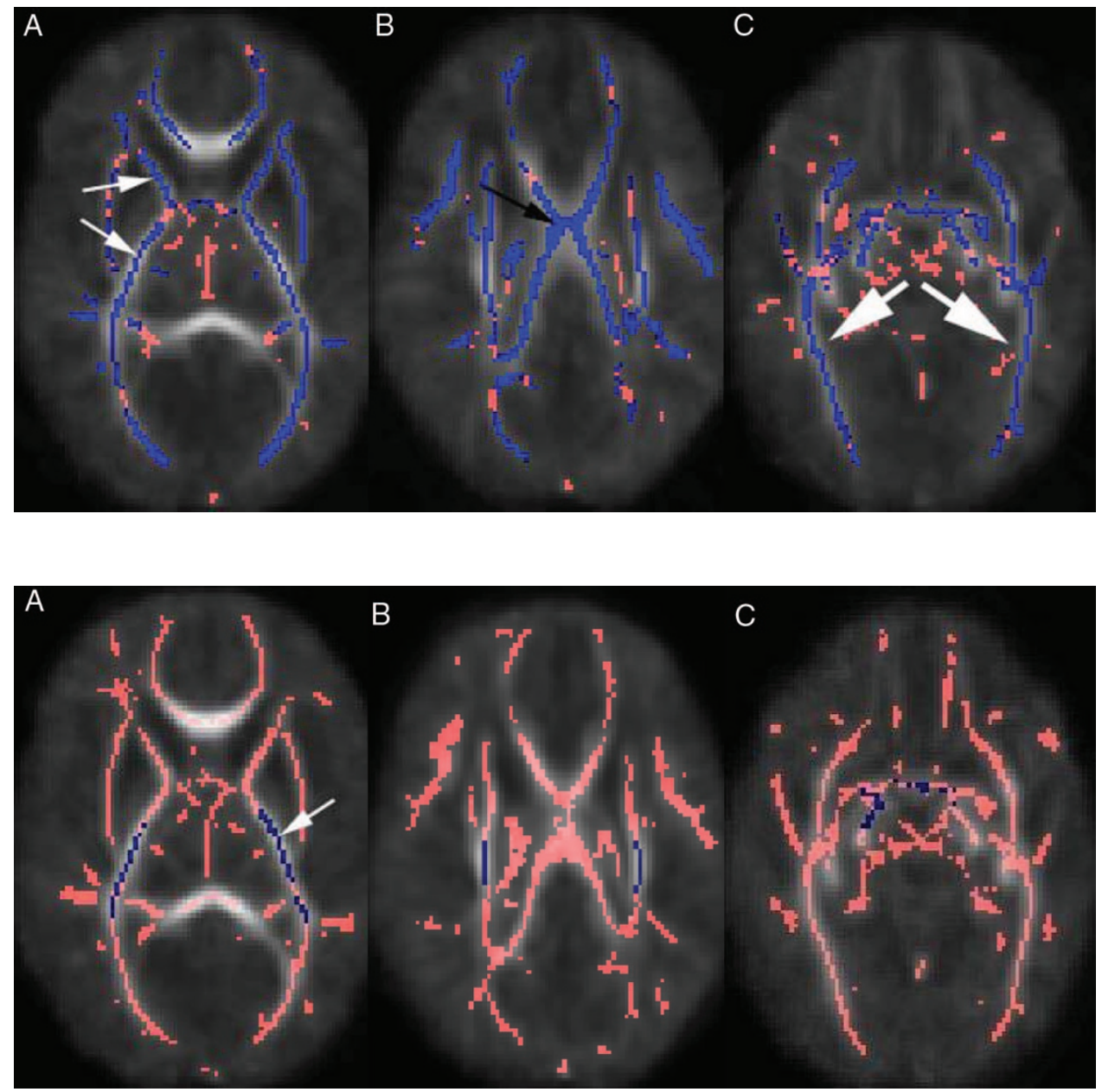

Figure 1. Axial images of mean FA of white matter tracts of all subjects (controls and HIE groups). The group mean FA skeleton is shown in pink. Areas where the noncooled group with HIE have statistically lower FA than the healthy term control group are shown in blue: $A$, internal and external capsules (small white arrows), $B$, body of the corpus callosum (black arrow), $C$, optic radiations (large white arrows).

Figure 2. Axial images of mean FA of white matter tracts of all subjects (controls and HIE groups). The group mean FA skeleton is shown in pink. The cooled group with HIE had statistically lower FA in the internal capsule (shown in blue indicated by small white arrow) compared with the healthy control group (A). $B$, shows white matter tracts at the level of corpus callosum and $(C)$ at the level of the optic radiations. 


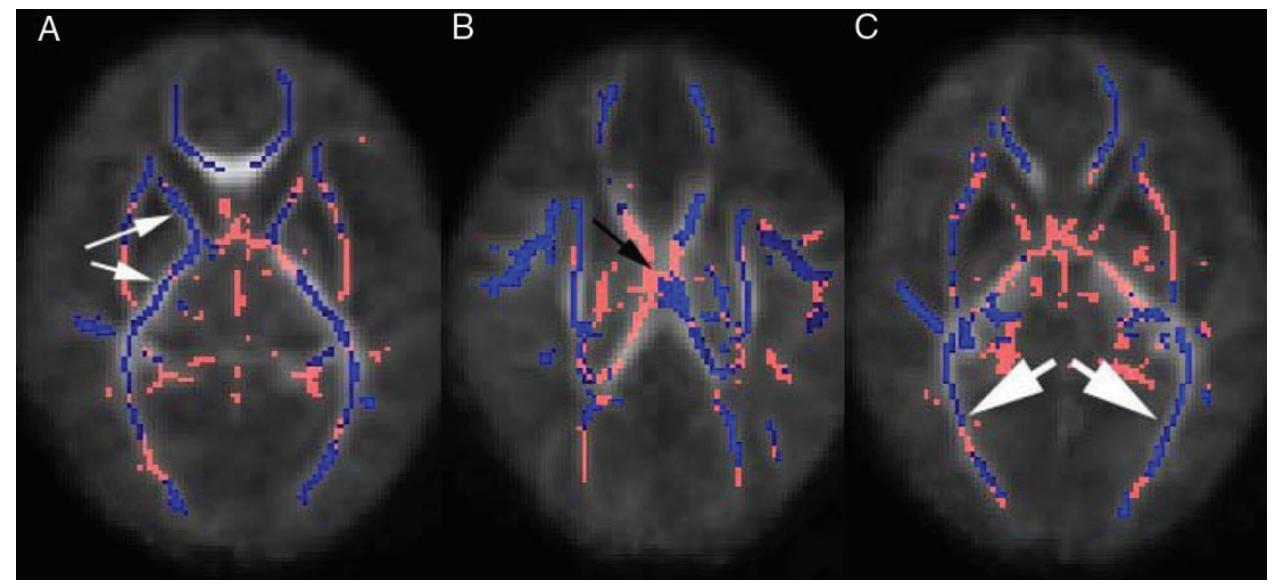

Figure 3. Axial images of mean FA of white matter tracts of all HIE subjects. The group mean FA skeleton is shown in pink. Areas where the noncooled group with HIE has a statistically lower FA than the cooled group are shown in blue: $A$, internal capsules and external capsules (small white arrows), $B$, body of the corpus callosum (black arrow), $C$, optic radiations (large white arrows).

Table 2. Mean FA values in white matter regions in infants with HIE

\begin{tabular}{llc}
\hline \multicolumn{1}{c}{ Region } & $\begin{array}{c}\text { Cooled mean } \\
\text { FA (standard } \\
\text { deviation) }\end{array}$ & $\begin{array}{c}\text { Noncooled mean } \\
\text { FA (standard } \\
\text { deviation) }\end{array}$ \\
\hline Right PLIC & $0.306(0.037)$ & $0.234(0.052)$ \\
Left PLIC & $0.352(0.031)$ & $0.295(0.047)$ \\
Right ALIC & $0.293(0.05)$ & $0.212(0.050)$ \\
Left ALIC & $0.207(0.035)$ & $0.146(0.042)$ \\
Corpus callosum & $0.266(0.048)$ & $0.196(0.038)$ \\
Right optic radiation & $0.353(0.05)$ & $0.273(0.058)$ \\
Left optic radiation & $0.335(0.034)$ & $0.269(0.036)$ \\
\hline
\end{tabular}

HIE, hypoxic ischemic encephalopathy; ALIC, anterior limb of internal capsule; PLIC, posterior limb of internal capsule.

titative measurements such as the apparent diffusion coefficient, and FA are altered in tissue damage $(14,15)$.

Using TBSS to analyze DTI data, comparing just 10 infants per group, we demonstrated widespread microstructural abnormalities in white matter tracts in full-term infants after perinatal asphyxia, which were reduced after therapeutic hypothermia. These data confirm that TBSS analysis of FA is a powerful tool for comparing white matter tissue integrity among patient groups and suggest that it is a suitable biomarker for early phase trials of novel neuroprotectants.

One important advantage of TBSS is the ability to investigate the entire white matter. Previous DTI studies in perinatal asphyxia encephalopathy have investigated prespecified ROI within the brain. This method is subjective and restricts the areas of investigation to those that are either easily identifiable or thought to be of greatest clinical significance and therefore may miss unexpected areas of injury. ROI-based analyses can be time consuming and often rely on investigator accuracy. These limitations make comparison of multiple brain regions across larger groups more difficult. In contrast, TBSS is a fully automated observer independent process that enables voxelwise and groupwise comparison of FA values across all the major white matter tracts common to our subjects without the need for prespecified ROI (18).

As with any group comparison tool, the registration of multiple images needs to be precise. The technique includes careful registration of each subjects' image onto a subjectspecific target image, and the use of a FA threshold that includes all the major white matter tracts but excludes areas of low mean FA such as gray matter or area of high inter-subject variability such as smaller more peripheral white matter tracts. Although visual assessment of MRI scans after hypoxic ischemic encephalopathy accurately identifies injury in central gray matter structures namely, the basal ganglia and thalami, this is not possible with TBSS because of the inherent low FA values of these structures, which is a limitation of our approach.

In summary, DTI analyzed by TBSS seems to provide a quantifiable, qualified biomarker of neurologic injury and treatment effect in hypoxic ischemic encephalopathy. The ability to compare voxelwise differences across groups of

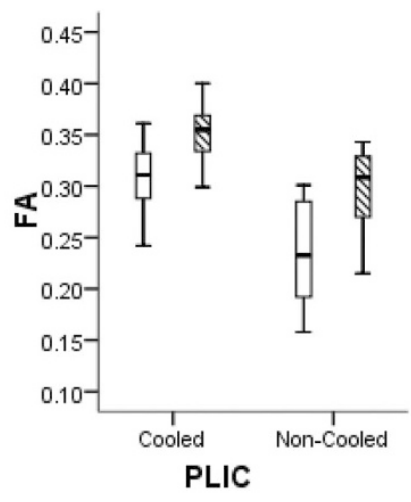

PLIC
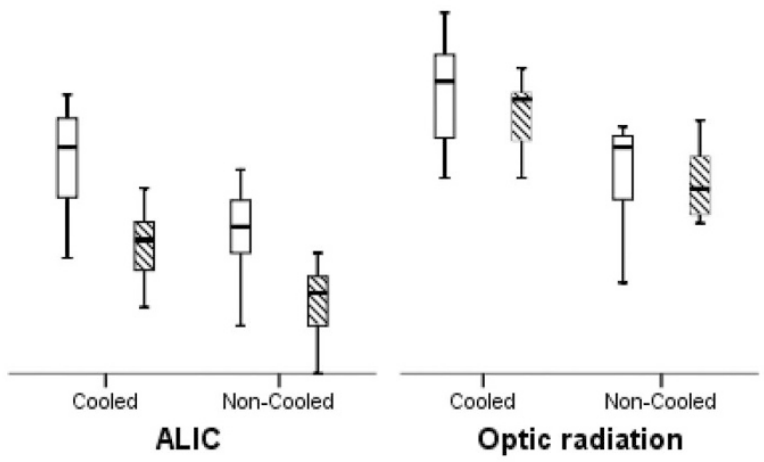

Figure 4. Comparison of mean FA values in cooled and noncooled infants. The minimum, first quartile, median, third quartile, and maximum are displayed. ALIC, anterior limb of internal capsule; PLIC, posterior limb of internal capsule. FA values from the right side $(\square)$ and from the left side $(\mathbf{\nabla})$. 
patients increases statistical power and allows trials with small group sizes suitable for proof of concept studies of potential neuroprotective interventions.

\section{REFERENCES}

1. Himmelmann K, Hagberg G, Beckung E, Hagberg B, Uvebrant P 2005 The changing panorama of cerebral palsy in Sweden. IX. Prevalence and origin in the birth-year period 1995-1998. Acta Paediatr 94:287-294

2. Gluckman PD, Wyatt JS, Azzopardi D, Ballard R, Edwards AD, Ferriero DM, Polin RA, Robertson CM, Thoresen M, Whitelaw A, Gunn AJ 2005 Selective head cooling with mild systemic hypothermia after neonatal encephalopathy: multicentre randomised trial. Lancet 365:663-670

3. Shankaran S, Laptook AR, Ehrenkranz RA, Tyson JE, McDonald SA, Donovan EF, Fanaroff AA, Poole WK, Wright LL, Higgins RD, Finer NN, Carlo WA, Duara S, Oh W, Cotten CM, Stevenson DK, Stoll BJ, Lemons JA, Guillet R, Jobe AH 2005 Whole-body hypothermia for neonates with hypoxic-ischemic encephalopathy. N Engl J Med 353:1574-1584

4. Azzopardi DV, Strohm B, Edwards AD, Dyet L, Halliday HL, Juszczak E, Kapellou O, Levene M, Marlow N, Porter E, Thoresen M, Whitelaw A, Brocklehurst P 2009 Moderate hypothermia to treat perinatal asphyxial encephalopathy. N Engl J Med 361:1349-1358

5. Ma D, Hossain M, Chow A, Arshad M, Battson RM, Sanders RD, Mehmet H, Edwards AD, Franks NP, Maze M 2005 Xenon and hypothermia combine to provide neuroprotection from neonatal asphyxia. Ann Neurol 58:182-193

6. Jouvet P, Cowan FM, Cox P, Lazda E, Rutherford MA, Wigglesworth J, Mehmet H, Edwards AD 1999 Reproducibility and accuracy of MR imaging of the brain after severe birth asphyxia. AJNR Am J Neuroradiol 20:1343-1348

7. Rutherford MA, Pennock JM, Counsell SJ, Mercuri E, Cowan FM, Dubowitz LM, Edwards AD 1998 Abnormal magnetic resonance signal in the internal capsule predicts poor neurodevelopmental outcome in infants with hypoxic-ischemic encephalopathy. Pediatrics 102:323-328

8. Rutherford M, Srinivasan L, Dyet L, Ward P, Allsop J, Counsell S, Cowan F 2006 Magnetic resonance imaging in perinatal brain injury: clinical presentation, lesions and outcome. Pediatr Radiol 36:582-592

9. Cowan F, Rutherford M, Groenendaal F, Eken P, Mercuri E, Bydder GM, Meiners LC, Dubowitz LM, de Vries LS 2003 Origin and timing of brain lesions in term infants with neonatal encephalopathy. Lancet 361:736-742
10. Chao CP, Zaleski CG, Patton AC 2006 Neonatal hypoxic-ischemic encephalopathy: multimodality imaging findings. Radiographics 26:S159-S172

11. Rutherford M, Ramenghi LA, Edwards AD, Brocklehurst P, Halliday H, Levene M, Strohm B, Thoresen M, Whitelaw A, Azzopardi D 2010 Assessment of brain tissue injury after moderate hypothermia in neonates with hypoxic-ischaemic encephalopathy: a nested substudy of a randomised controlled trial. Lancet Neurol 9:39-45

12. Pierpaoli C, Jezzard P, Basser PJ, Barnett A, Di Chiro G 1996 Diffusion tensor MR imaging of the human brain. Radiology 201:637-648

13. Basser PJ, Pierpaoli C 1996 Microstructural and physiological features of tissues elucidated by quantitative-diffusion-tensor MRI. J Magn Reson B 111:209-219

14. Ward P, Counsell S, Allsop J, Cowan F, Shen Y, Edwards D, Rutherford M 2006 Reduced fractional anisotropy on diffusion tensor magnetic resonance imaging after hypoxic-ischemic encephalopathy. Pediatrics 117:e619-e630

15. Hunt RW, Neil JJ, Coleman LT, Kean MJ, Inder TE 2004 Apparent diffusion coefficient in the posterior limb of the internal capsule predicts outcome after perinatal asphyxia. Pediatrics 114:999-1003

16. Rutherford M, Counsell S, Allsop J, Boardman J, Kapellou O, Larkman D, Hajna J, Edwards D, Cowan F 2004 Diffusion-weighted magnetic resonance imaging in term perinatal brain injury: a comparison with site of lesion and time from birth Pediatrics 114:1004-1014

17. Dag Y, Firat AK, Karakas HM, Alkan A, Yakinci C, Erdem G 2006 Clinical outcomes of neonatal hypoxic ischemic encephalopathy evaluated with diffusionweighted magnetic resonance imaging. Diagn Interv Radiol 12:109-114

18. Smith SM, Jenkinson M, Johansen-Berg H, Rueckert D, Nichols TE, Mackay CE, Watkins KE, Ciccarelli O, Cader MZ, Matthews PM, Behrens TE 2006 Tract-based spatial statistics: voxelwise analysis of multi-subject diffusion data. Neuroimage 31:1487-1505

19. Thayyil S, Chandrasekaram M, Taylor A, Cady E, Chong K, Murad S, Omar R, Robertson NJ 2010 Cerebral magnetic resonance biomarkers for predicting longterm neurodevelopmental outcome following neonatal encephalopathy: a metaanalysis. Pediatrics 125:e382-e395

20. Thoresen M, Penrice J, Lorek A, Cady EB, Wylezinska M, Kirkbride V, Cooper CE, Brown GC, Edwards AD, Wyatt JS 1995 Mild hypothermia after severe transient hypoxia-ischemia ameliorates delayed cerebral energy failure in the newborn piglet. Pediatr Res 37:667-670

21. Blumberg RM, Cady EB, Wigglesworth JS, McKenzie JE, Edwards AD 1997 Relation between delayed impairment of cerebral energy metabolism and infarction following transient focal hypoxia-ischaemia in the developing brain. Exp Brain Res 113:130-137 